\title{
Proposal for an Optomechanical Bell Test
}

\author{
V. Caprara Vivoli, ${ }^{1}$ T. Barnea, ${ }^{1}$ C. Galland, ${ }^{2}$ and N. Sangouard ${ }^{3}$ \\ ${ }^{1}$ Group of Applied Physics, University of Geneva, CH-1211 Geneva 4, Switzerland \\ ${ }^{2}$ Ecole Polytechnique Federale de Lausanne (EPFL), CH-1015 Lausanne, Switzerland \\ ${ }^{3}$ Department of Physics, University of Basel, CH-4056 Basel, Switzerland
}

(Received 26 June 2015; revised manuscript received 9 October 2015; published 18 February 2016)

\begin{abstract}
Photons of a laser beam driving the upper motional sideband of an optomechanical cavity can decay into photon-phonon pairs by means of an optomechanical parametric process. The phononic state can subsequently be mapped to a photonic state by exciting the lower sideband, hence creating photon-photon pairs out of an optomechanical system. Here we show that these pairs can violate a Bell inequality when they are measured with photon counting techniques preceded by small displacement operations in phase space. The consequence of such a violation as well as the experimental requirements are intensively discussed.
\end{abstract}

DOI: 10.1103/PhysRevLett.116.070405

Introduction.-Cavity optomechanics, which describes a mechanical oscillator controlled by an electromagnetic cavity mode via a generalized radiation pressure force, is the subject of intense research [1-3]. Most recent progress includes the cooling of mechanical oscillators down to the ground state [4-6], the readout of the mechanical position with a readout imprecision below the standard quantum limit [7] as well as optomechanical squeezing [8,9] and entanglement [10]. Reciprocally, the mechanical degrees of freedom can be used to control the cavity light, e.g., for fast and slow light $[11,12]$, frequency conversions [13,14], squeezing [15], and information storage in long-lived mechanical oscillations $[10,16]$.

Optomechanical systems are also envisioned as test benches for physical theories [17-23]. As a step in this direction, quantum correlations between light and mechanics have been observed recently [10]. In this experiment, quantum features have been detected through an entanglement witness where one assumes that the measurement devices are well characterized and where quantum theory is used to predict the results of these measurements on separable states. It is interesting to wonder whether the nonclassical behavior of optomechanical systems can be certified outside of the quantum formalism, i.e., from a Bell test [24]. This is particularly relevant to test postquantum theories including explicit collapse models [25-28], where the assumption that the system behaves quantum mechanically may be questionable [29].

In this Letter, we show how to perform such a Bell test in the experimentally relevant weak-optomechanical coupling and sideband-resolved regime. Our proposal, which starts with a mechanical oscillator close to its ground state, consists of two steps. First, the optomechanical system is excited by a laser tuned to the upper motional sideband of the cavity to create photon-phonon pairs via optomechanical parametric conversion. Second, a laser resonant with the lower sideband is used to map the phononic state to the cavity field. The correlations between the photons generated at the cavity frequency during the first and second steps are then analyzed by photon counting preceded by small displacement operations in phase space. We show that they violate the Bell-CHSH (Clauser-Horne-ShimonyHolt) inequality [30], revealing that the optomechanical state is nonlocal, i.e., provides stronger correlations than entanglement [31]. This claim is device independent, i.e., holds without assumptions on the dimension of the underlying Hilbert space or on the precise alignment of the measurement settings [32]. While several requirements are challenging to meet in practice, our proposal can be seen as a natural extension of ongoing experiments [33] performing photon counting in optomechanical cavities.

Principle of the optomechanical Bell test.-The basic principle is inspired by Refs. [34,35] and is represented in Fig. 1. We use two laser pulses driving either the upper or the lower optomechanical sideband, at frequency $\omega_{ \pm}$, which is the sum or the difference of the cavity $\left(\omega_{c}\right)$ and the mechanical $\left(\Omega_{m}\right)$ frequencies. The optomechanical Hamiltonian includes $H_{0}=\hbar \omega_{c} a^{\dagger} a+\hbar \Omega_{m} b^{\dagger} b$, the uncoupled cavity and mechanical systems with respective bosonic operators $a$ and $b, H_{\mathrm{OM}}=-\hbar g_{0} a^{\dagger} a\left(b^{\dagger}+b\right)$, the optomechanical interaction with $g_{0}$ the optomechanical coupling, and $H_{l}=\hbar\left(s_{ \pm}^{*} e^{i \omega_{ \pm} t} a+s_{ \pm} e^{-i \omega_{ \pm} t} a^{\dagger}\right)$, the driving laser with $\left|s_{ \pm}\right|=\sqrt{\kappa P_{ \pm} / \hbar \omega_{ \pm}}, P_{ \pm}$being the laser power and $\kappa$ the cavity decay rate (assuming that the intracavity loss is negligible). In the interaction picture, the weak coupling limit $g_{0} \ll \kappa$ and the resolved sideband regime $\kappa \ll \Omega_{m}$, the dynamics are given by a set of Langevin equations,

$$
\begin{aligned}
& \frac{d a}{d t}=\frac{i}{\hbar}\left[H_{ \pm}, a\right]-\frac{\kappa}{2} a+\sqrt{\kappa} a_{\mathrm{in}}, \\
& \frac{d b}{d t}=\frac{i}{\hbar}\left[H_{ \pm}, b\right]-\frac{\gamma}{2} b+\sqrt{\gamma} b_{\mathrm{in}},
\end{aligned}
$$




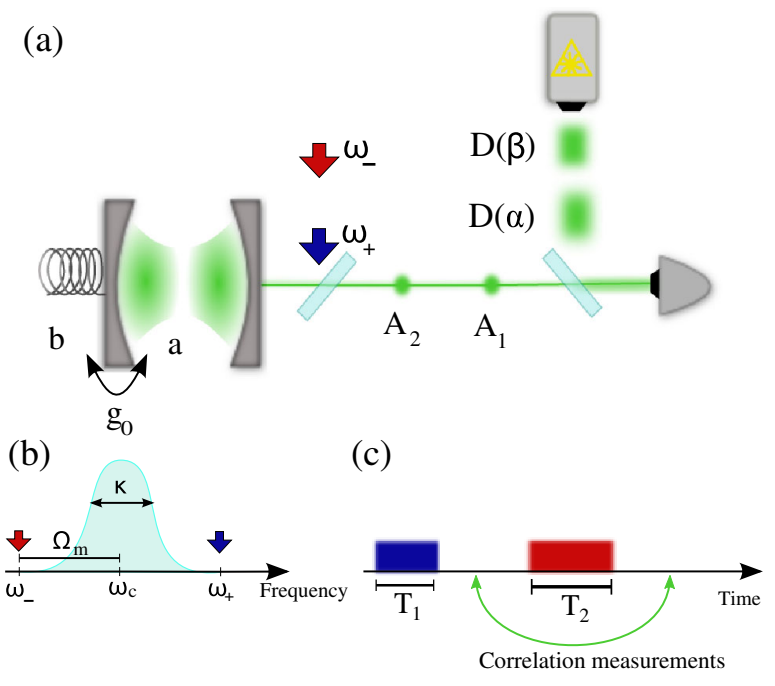

FIG. 1. (a) Principle of the proposed Bell test. Starting with the mechanical system in its motional ground state, a first laser excites the blue detuned sideband to create correlated photonphonon pairs. A second laser then drives the red detuned sideband to coherently convert the phonons into photons. The resulting photons pairs (mode $A_{1}$ and $A_{2}$ ) are analyzed using a photon detector preceded by a displacement in phase space. (b) Cavity linewidth $\kappa$ and its two sidebands $\left(\omega_{+}\right)$detuned from the cavity frequency $\omega_{c}$ by the mechanical frequency $\left(\omega_{m}\right)$. (c) Pulse sequence in time. The first laser resonantly excites the blue sideband while the second laser is resonant with the red sideband.

with the linearized Hamiltonians $H_{+}=-g_{+} \hbar a^{\dagger} b^{\dagger}+$ H.c. for a blue detuned drive and $H_{-}=-g_{-} \hbar a^{\dagger} b+$ H.c. for a red detuned drive. $g_{ \pm}$are the effective optomechanical coupling rates enhanced by the intracavity photon number $g_{ \pm}=g_{0} \sqrt{n_{ \pm}}=\left(\kappa P_{ \pm} / \hbar \omega_{c}\left(\Omega_{m}^{2}+\kappa^{2} / 4\right)\right) \cdot a_{\text {in }}$ is the vacuum noise entering the cavity. We assume that the laser is shot-noise limited; hence, it does not add contributions to the input noise. $b_{\text {in }}$ is the thermal noise from a phonon bath at temperature $T_{\text {bath }}$ and mean occupation number $n_{\text {th }}=\left(k_{B} T_{\text {bath }} / \hbar \Omega_{m}\right)$. In the following treatment, we neglect the mechanical decay which is well justified for time scales smaller than the thermal decoherence time $1 / \gamma n_{\text {th }}, \gamma$ being the coupling rate between the mechanical oscillator and the thermal bath.

Consider first the case where the mechanics is driven by a blue detuned laser. In the regime $g_{+} \ll \kappa$, the cavity mode can be adiabatically eliminated and Eq. (1) leads to $a_{1}=$ $(2 / \kappa)\left(i g_{+} b^{\dagger}+\sqrt{\kappa} a_{1, \text { in }}\right)$ (the subscript on the cavity field operators is used to recall that we are considering the first step). Further, introducing the input-output relation, $a_{1, \text { out }}=-a_{1, \text { in }}+\sqrt{\kappa} a_{1}$, we obtain

$$
\begin{aligned}
& a_{1, \mathrm{out}}=a_{1, \mathrm{in}}+i \sqrt{2 \bar{g}_{+}} b^{\dagger}, \\
& \frac{d b}{d t}=\bar{g}_{+} b+i \sqrt{2 \bar{g}_{+}} a_{1, \mathrm{in}}^{\dagger},
\end{aligned}
$$

where $\bar{g}_{+}=\left(2 g_{+}^{2} / \kappa\right)$. To solve these coupled equations, we follow Hofer et al. [35] and introduce the temporal modes $A_{1, \text { in }}(t)=\sqrt{\left(2 \bar{g}_{+} / 1-e^{-2 \bar{g}_{+} t}\right)} \int_{0}^{t} d t^{\prime} e^{-\bar{g}_{+} t^{\prime}} a_{1, \text { in }}\left(t^{\prime}\right), A_{1, \text { out }}(t)=$ $\sqrt{\left(2 \bar{g}_{+} / e^{2 \bar{g}_{+} t}-1\right)} \int_{0}^{t} d t^{\prime} e^{\bar{g}_{+} t^{\prime}} a_{1, \text { out }}\left(t^{\prime}\right)$. The solutions of Eqs. (3) and (4) take the following simple forms: $A_{1, \text { out }}(t)=e^{\bar{g}_{+} t} A_{1, \text { in }}(t)+i \sqrt{e^{2 \bar{g}_{+} t}-1} b^{\dagger}(0), b(t)=e^{\bar{g}_{+} t} b(0)+$ $i \sqrt{e^{2 \bar{g}_{+} t}-1} A_{1, \text { in }}^{\dagger}(t)$. These solutions can be rewritten as $A_{1, \text { out }}=\tilde{U}_{1}^{\dagger}(t) A_{1, \text { in }} \tilde{U}_{1}(t)$ and $b(t)=\tilde{U}_{1}^{\dagger}(t) b(0) \tilde{U}_{1}(t)$, where the propagator is given by

$$
\begin{aligned}
\tilde{U}_{1}(t)= & e^{i \sqrt{1-e^{-2 \bar{g}+t}} A_{1, \mathrm{in}}^{\dagger} b^{\dagger}} \\
& \times e^{\bar{g}_{+} t\left(-1-A_{1, \mathrm{n}}^{\dagger} A_{1, \mathrm{in}}-b^{\dagger} b\right)} e^{i \sqrt{1-e^{-2 \bar{g}+t}} A_{1, \mathrm{in}} b} .
\end{aligned}
$$

When applied on the vacuum, this propagator leads to the creation of photon-phonon pairs where the number of photons equals the number of phonons, each of them following a thermal statistics with mean excitation number $e^{2 \bar{g}_{+} t}-1$.

Now consider the case where the mechanics is driven by a red detuned laser; i.e., the dynamics is given by the beam splitter Hamiltonian $H_{-}$. Following the same procedure as before, Eqs. (3) and (4) become

$$
\begin{gathered}
a_{2, \mathrm{out}}=a_{2, \mathrm{in}}+i \sqrt{2 \bar{g}_{-}} b, \\
\frac{d b}{d t}=-\bar{g}_{-} b+i \sqrt{2 \bar{g}_{-}} a_{2, \mathrm{in}},
\end{gathered}
$$

where $\quad \bar{g}_{-}=\left(2 g_{-}^{2} / \kappa\right)$. Introducing the modes $A_{2, \text { in }}(t)=\sqrt{\left(2 \bar{g}_{-} / e^{2 \bar{g}_{-} t}-1\right)} \int_{0}^{t} d t^{\prime} e^{\bar{g}_{-} t^{\prime}} a_{2, \text { in }}\left(t^{\prime}\right), \quad A_{2, \text { out }}(t)=$ $\sqrt{\left(2 \bar{g}_{-} / 1-e^{-2 \bar{g}_{-} t}\right)} \int_{0}^{t} d t^{\prime} e^{-\bar{g}_{-} t^{\prime}} a_{2, \text { out }}\left(t^{\prime}\right)$ leads to the simple expression for the solutions of Eqs. (6) and (7) at a time $t$ after the beginning of the red detuned pulse $A_{2 \text {,out }}(t)=$ $e^{-\bar{g}_{-} t} A_{2, \text { in }}(t)+i \sqrt{1-e^{-2 \bar{g}_{-} t}} b(0), \quad b(t)=e^{-\bar{g}_{-} t} b(0)+$ $i \sqrt{1-e^{-2 \bar{g}_{-} t}} A_{2 \text {,in }}(t)$. These solutions can be rewritten as $A_{2, \text { out }}=\tilde{U}_{2}^{\dagger}(t) A_{2, \text { in }} \tilde{U}_{2}(t) \quad$ and $\quad b(t)=\tilde{U}_{2}^{\dagger}(t) b(0) \tilde{U}_{2}(t)$, where the propagator is given by

$$
\begin{aligned}
\tilde{U}_{2}(t)= & e^{i \sqrt{e^{2 \bar{g}-t}-1} A_{2, \mathrm{in}} b^{\dagger}} \\
& \times e^{-\bar{g}_{-} t\left(A_{2, \mathrm{in}}^{\dagger} A_{2, \mathrm{in}}-b^{\dagger} b\right)} e^{i \sqrt{e^{2 \bar{g}-t}-1} A_{2, \mathrm{in}}^{\dagger} b} .
\end{aligned}
$$

This corresponds to a process converting a phonon into a photon with probability $1-e^{-2 \bar{g}_{-} t}$.

Now consider an initial state where both optical modes $A_{1}$ and $A_{2}$ are empty and where the mechanics is prepared in its ground state. Switching on the blue detuned laser for a time $T_{1}$, then the red detuned laser for a time $T_{2}$, leads to a photon-photon state in mode $A_{1, \text { out }}, A_{2, \text { out }}$ given by $\rho_{A_{1}, A_{2}}=$ $\operatorname{Tr}_{b} \tilde{U}_{2}\left(T_{2}\right) \tilde{U}_{1}\left(T_{1}\right)|0,0,0\rangle_{A_{1, \mathrm{in},}, A_{2, \mathrm{in}}, b}\langle 0,0,0| \tilde{U}_{1}^{\dagger}\left(T_{1}\right) \tilde{U}_{2}^{\dagger}\left(T_{2}\right)$. In the ideal limit $\bar{g}_{-} T_{2} \rightarrow+\infty$, the phonon-photon mapping is perfect and the state $\rho_{A_{1}, A_{2}}$ corresponds to a 
two-mode squeezed vacuum. In the general case where $\bar{g}_{-} T_{2}$ has a finite value, $\rho_{A_{1}, A_{2}}$ still corresponds to a squeezed vacuum but where the mode $A_{2}$ undergoes loss. This loss can be modeled by a beam splitter with a transmission $T=1-e^{-2 \bar{g}_{-} t}$. The next section shows how to reveal the nonlocal content of such a state.

In order to test a Bell inequality with the modes $A_{1, \text { out }}$, $A_{2, \text { out }}$ (the subscript "out" is omitted below), we consider a single-photon detector-which does not resolve the photon number-combined with a displacement operation $D(\alpha)$. We associate the outcome $+1(-1)$ to the absence of detection (to the detection of at least one photon) at least one photon. In the subspace composed of the vacuum and the single-photon Fock state, such a measurement corresponds exactly to the observable $\sigma_{z}$ for $\alpha=0$, while for $\alpha=1$ $(\alpha=i)$ it is a noisy $\sigma_{x}\left(\sigma_{y}\right)$ [36]. The potential of such measurements for nonlocality detection has been highlighted in Refs. [37,38], for example. Reference [39] has also shown how they can be used for Bell tests in photonic experiments where two-mode squeezed states are produced through spontaneous parametric down-conversion. More recently, they have been used to reveal genuine path entanglement [40]. Further note that a displacement is easy to implement in practice as it requires a coherent state and an unbalanced beam splitter only [41].

The joint probability $P\left(+1+1 \mid \alpha_{1} \alpha_{2}\right)$ to get the outcomes +1 for both $A_{1}$ and $A_{2}$ when they are analyzed with photon counting with efficiency $\eta$ preceded by displacements with amplitude $\alpha_{1}$ and $\alpha_{2}$ for $A_{1}$ and $A_{2}$, respectively, is given by $P\left(+1+1 \mid \alpha_{1} \alpha_{2}\right)=\operatorname{Tr}\left[\rho_{A_{1}, A_{2}} \mathcal{O}_{\eta}\left(\alpha_{1}, A_{1}\right) \otimes\right.$ $\left.\mathcal{O}_{\eta}\left(\alpha_{2}, A_{2}\right)\right]$, where $\mathcal{O}_{\eta}\left(\alpha_{i}, A_{i}\right)=D^{\dagger}\left(\alpha_{i}\right)(1-\eta)^{A_{i}^{\dagger} A_{i}} D\left(\alpha_{i}\right)$. Such a probability can be computed easily by noting that loss and displacement can be commuted by changing the amplitude of the displacement. In particular, $P(+1+$ $\left.1 \mid \alpha_{1} \alpha_{2}\right)=\operatorname{Tr}\left[\bar{\rho}_{A_{1}, A_{2}} \mathcal{O}_{\eta}\left(\alpha_{1}, A_{1}\right) \otimes \mathcal{O}_{\eta^{\prime}}\left(\alpha_{2} \sqrt{T}, A_{2}\right)\right]$, where $\eta^{\prime}=\eta T$, and $\bar{\rho}_{A_{1}, A_{2}}=(1-p) e^{-\sqrt{p} A_{1}^{\dagger} A_{2}^{\dagger}}|0\rangle_{A_{1}, A_{2}}\langle 0| e^{-\sqrt{p} A_{1} A_{2}}$ is simply the two-mode squeezed vacuum. $(1-p=$ $e^{-2 \bar{g}_{+} T_{1}}$ is the probability that both modes are empty.) We find

$P\left(+1+1 \mid \alpha_{1} \alpha_{2}\right)=\frac{(1-p)}{1-p(1-\eta)\left(1-\eta^{\prime}\right)} e^{-\left\{\eta\left|\alpha_{1}\right|^{2}\left[1-\left(1-\eta^{\prime}\right) p\right]+\eta^{\prime}\left|\alpha_{2}\right|^{2} T[1-(1-\eta) p]\right\} /\left[1-p(1-\eta)\left(1-\eta^{\prime}\right)\right]} e^{\eta \eta^{\prime} \sqrt{p}\left(\alpha_{1}^{*} \alpha_{2}^{*}+\alpha_{1} \alpha_{2}\right) \sqrt{T} /\left[1-p(1-\eta)\left(1-\eta^{\prime}\right)\right]}$.

Together with the marginals

$$
\begin{aligned}
& P\left(+1 \mid \alpha_{1}\right)=\frac{(1-p)}{1-p(1-\eta)} \times e^{-\eta(1-p)\left|\alpha_{1}\right|^{2} /[1-p(1-\eta)]}, \\
& P\left(+1 \mid \alpha_{2}\right)=\frac{(1-p)}{1-p\left(1-\eta^{\prime}\right)} \times e^{-\eta^{\prime}(1-p)\left|\alpha_{2}\right|^{2} T /\left[1-p\left(1-\eta^{\prime}\right)\right]},
\end{aligned}
$$

we get the explicit value of the correlator $E^{\alpha_{1}, \alpha_{2}}=$ $1-2\left[P\left(+1 \mid \alpha_{1}\right)+P\left(+1 \mid \alpha_{2}\right)\right]+4 P\left(+1+1 \mid \alpha_{1} \alpha_{2}\right)$ to test the Bell-CHSH inequality, $\mathrm{CHSH}=\mid E^{\alpha_{1}, \alpha_{2}}+E^{\alpha_{1}^{\prime}, \alpha_{2}}+E^{\alpha_{1}, \alpha_{2}^{\prime}}-$ $E^{\alpha_{1}^{\prime}, \alpha_{2}^{\prime}} \mid \leq 2$, which holds for any local hidden-variable model.

Figure 2 shows the $\mathrm{CHSH}$ values obtained from the optimization over the measurement settings $\alpha_{i}, \alpha_{i}^{\prime}, i \in[1,2]$ as a function of the photon-phonon mapping efficiency $T=1-e^{-2 \bar{g}_{-} t}$ for various detection efficiencies $\eta$. For high enough $T$ and $\eta$, we see that the CHSH inequality is violated, hence, showing that the correlations of modes $A_{1}$ and $A_{2}$ cannot be reproduced by local hidden-variable theories.

In the above discussion, we have assumed that the mechanical system is prepared in its ground state. In the more general case it is in a thermal state with mean occupation number $n_{0}$; the expressions of the joint probability $P\left(+1+1 \mid \alpha_{1} \alpha_{2}\right)$ and the marginals $P\left(+1 \mid \alpha_{i}\right)$ can be derived as before; cf. Ref. [42], part I. The CHSH values resulting from the optimization over the measurement settings are given in Fig. 3 as a function of the phononphoton mapping efficiency for various mean mechanical occupation numbers assuming unit detection efficiencies. A substantial violation can be obtained if $n_{0} \ll 1$.

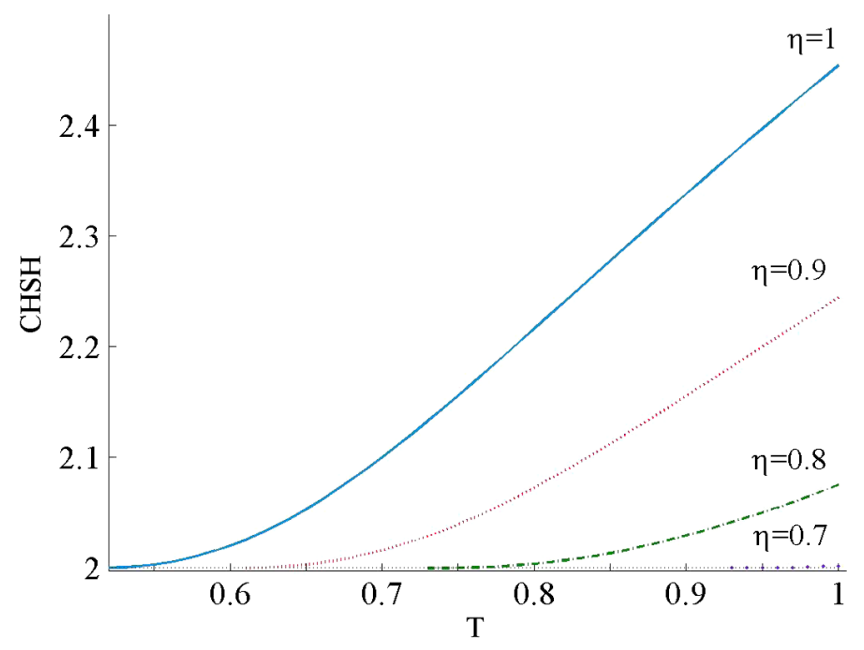

FIG. 2. CHSH values optimized over the measurement settings $\left(\alpha_{1}, \alpha_{2}\right)$ as a function of the optomechanical mapping efficiency $\left(T=1-e^{-2 \bar{g}_{-} t}\right)$ for various detection efficiencies $\eta$. The $\mathrm{CHSH}$ value is larger than the local bound 2 for unit detection efficiencies when $T \geq 52 \%$, while for unit phonon-photon mapping efficiency, $\eta \geq 66.8 \%$ is required. 


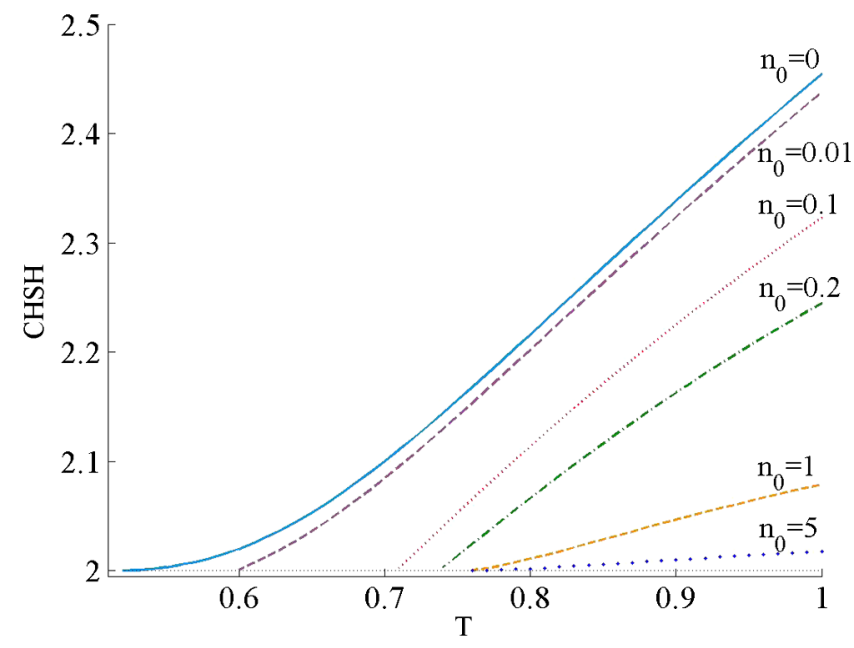

FIG. 3. CHSH values as a function of the optomechanical mapping efficiency $\left(T=1-e^{-2 \bar{g}_{-} t}\right)$ for various mechanical occupation numbers $\left(n_{0}\right)$ assuming unit detection efficiencies $(\eta=1)$.

Feasibility.-In this paragraph, we discuss the experimental feasibility of the proposed Bell test in detail. The requirements for detecting nonlocality are (i) sideband regime $\kappa \ll \Omega_{m}$, (ii) weak coupling regime $g_{0} \ll \kappa$, (iii) ground state cooling $n_{0} \ll 1$ and negligible mechanical decoherence during the durations of blue and red detuned laser pulses $T_{1}+T_{2} \ll\left(\gamma n_{\mathrm{th}}\right)^{-1}$. Given that $n_{\mathrm{th}} \propto \Omega_{m}^{-1}$, these conditions are easier to meet with high $Q$ and high frequency $\Omega_{m}$ oscillators. While superconducting microwave optomechanical cavity systems are promising [44], we focus on an implementation of our Bell test with a photonic crystal nanobeam resonator $[6,45,46]$ that distinguishes itself by a very high mechanical frequency $\Omega_{m} / 2 \pi=5 \mathrm{GHz}$. This frequency together with its optical linewidth $\kappa / 2 \pi<1 \mathrm{GHz}$ places this resonator in the resolved sideband regime [45]. The optomechanical coupling rate is large $g_{0} / 2 \pi \approx 1 \mathrm{MHz}$ [45], and mechanical coherence times of the order of 10-100 $\mu$ s are expected at $4 \mathrm{~K}$ and below [45,47]. With a bath temperature $T_{\text {bath }} \approx 1.6 \mathrm{~K}$, an initial occupancy of $n_{0}=0.01$ can be achieved in $100 \mathrm{~ns}$ of sideband cooling with 1000 (intracavity) photons corresponding to a peak laser power of $150 \mu \mathrm{W}$ [34]. For almost squared pulses with rising time of the order of $1 \mathrm{~ns}$, we get pure and noiseless emissions as expected from two-mode squeeezed states [34]. For $T_{1}=25 \mathrm{~ns}$ and $T_{2}=50 \mathrm{~ns}$, we find $\mathrm{CHSH}=2.19$ assuming $\eta=90 \%$ detection efficiency, fixing $n_{-}=250$, and optimizing the CHSH value over $n_{+}=75$, and over the measurement settings. As the present scheme relies on the ability to accurately and repeatedly address the optomechanical sidebands, a continuous monitoring is required to correct the unavoidable broadband frequency noise of the cavity. Furthermore, the scattered photon pairs have to be spectrally filtered from the pump lasers. To account for an imperfect filtering of both the cavity locking and pump lasers, we include a background noise in the detection that we model as dark counts. We found that the $\mathrm{CHSH}$ violation $(\mathrm{CHSH}=2.19)$ is unchanged if the dark count probability is of $\leq 10^{-3}$. This sets the constraints on the quality of the filtering processes once the intrinsic noise of the detector is determined. Note that for state-of-the-artdetectors, the noise can be of the order of $10^{-7}$ or $10^{-8}$ for detection windows of a few tens of ns.

Perspectives.-Our results show how optomechanical systems can be used to test a Bell inequality. They provide an attractive perspective for the experiment reported in Ref. [33], where a mechanical system is combined with photon counting techniques. Achieving high overall detection efficiencies is facilitated by photons emitted in a well-defined spatial mode which may be coupled into a single-mode fiber with a very high efficiency. Moreover, the wavelength of photons at $1550 \mathrm{~nm}$ is an appealing asset to perform the Bell test between distant locations, i.e., to close the locality loophole; see Ref. [42] part II. We also note that our results can find interesting perspectives in quantum memory experiments. In the context of light storage, off-resonant Raman scattering can be used to create photon-spin wave pairs in atomic ensembles [48]. The spin-wave state can then be mapped to photons using a resonant Raman process-this mapping is made very efficient thanks to a collective emission. Since the resulting photon-photon state is analog to the one described in this Letter, the Bell test that we propose would allow one to certify that the memory operates in the quantum regime.

We thank K. Hammerer and S. Hofer for having pointed out the interest of an optomechanical Bell test at an early stage of this work. This work was supported by the Swiss National Science Foundation (SNSF), through Grant No. PP00P2-150579, the Swiss NCCR QSIT, and a SNSF Ambizione Fellowship, as well as by the Swiss State Secretariat for Education and Research through the COST Action MP1006.

Note added-While our proposal focuses on optomechanical systems, a Bell test can be performed with electromechanical systems. Recently, we became aware of a proposal along this line by Hofer et al. [49].

[1] T. J. Kippenberg and K. J. Vahala, Science 321, 1172 (2008).

[2] P. Meystre, Ann. Phys. (Berlin) 525, 215 (2013).

[3] M. Aspelmeyer, T. J. Kippenberg, and F. Marquardt, Rev. Mod. Phys. 86, 1391 (2014).

[4] A. D. O'Connell, M. Hofheinz, M. Ansmann, R. C. Bial-czak, M. Lenander, E. Lucero, M. Neeley, D. Sank, H. Wang, M. Weides, J. Wenner, J. M. Martinis, and A. N. Cleland, Nature (London) 464, 697 (2010). 
[5] J. D. Teufel, T. Donner, D. Li, J. W. Harlow, M. S. Allman, K. Cicak, A. J. Sirois, J. D. Whittaker, K. W. Lehnert, and R. W. Simmonds, Nature (London) 475, 359 (2011).

[6] J. Chan, T. P. M. Alegre, A. H. Safavi-Naeini, J. T. Hill, A. Krause, S. Groblacher, M. Aspelmeyer, and O. Painter, Nature (London) 478, 89 (2011).

[7] G. Anetsberger, E. Gavartin, O. Arcizet, Q. P. Unterreithmeier, E. M. Weig, M. L. Gorodetsky, J.P. Kotthaus, and T. J. Kippenberg, Phys. Rev. A 82, 061804 (2010).

[8] E. E. Wollman, C. U. Lei, A. J. Weinstein, J. Suh, A. Kronwald, F. Marquart, A. A. Clerk, and K. C. Schwab, Science 349, 952 (2015).

[9] J.-M. Pirkkalainen, E. Damskagg, M. Brandt, F. Massel, and M. A. Sillanpaa, Phys. Rev. Lett. 115, 243601 (2015).

[10] T. A. Palomaki, J. D. Teufel, R. W. Simmonds, and K. W. Lehnert, Science 342, 710 (2013).

[11] A. H. Safavi-Naeini, T. P. M. Alegre, J. Chan, M. Eichenfield, M. Winger, Q. Lin, J. T. Hill, D. E. Chang, and O. Painter, Nature (London) 472, 69 (2011).

[12] X. Zhou, F. Hocke, A. Schliesser, A. Marx, H. Huebl, R. Gross, and T. J. Kippenberg, Nat. Phys. 9, 179 (2013).

[13] J. Bochmann, A. Vainsencher, D. D. Awschalom, and A. N. Cleland, Nat. Phys. 9, 712 (2013).

[14] R. W. Andrews, R. W. Peterson, T. P. Purdy, K. Cicak, R. W. Simmonds, C. A. Regal, and K. W. Lehnert, Nat. Phys. 10, 321 (2014).

[15] A. H. Safavi-Naeini, S. Groeblacher, J. T. Hill, J. Chan, M. Aspelmeyer, and O. Painter, Nature (London) 500, 185 (2013).

[16] V. Fiore, Y. Yang, M. C. Kuzyk, R. Barbour, L. Tian, and H. Wang, Phys. Rev. Lett. 107, 133601 (2011).

[17] S. Bose, K. Jacobs, and P. L. Knight, Phys. Rev. A 59, 3204 (1999).

[18] W. Marshall, C. Simon, R. Penrose, and D. Bouwmeester, Phys. Rev. Lett. 91, 130401 (2003).

[19] P. Sekatski, M. Aspelmeyer, and N. Sangouard, Phys. Rev. Lett. 112, 080502 (2014).

[20] R. Ghobadi, S. Kumar, B. Pepper, D. Bouwmeester, A. I. Lvovsky, and C. Simon, Phys. Rev. Lett. 112, 080503 (2014).

[21] M. Bahrami, M. Paternostro, A. Bassi, and H. Ulbricht, Phys. Rev. Lett. 112, 210404 (2014).

[22] S. Nimmrichter, K. Hornberger, and K. Hammerer, Phys. Rev. Lett. 113, 020405 (2014).

[23] L. Diosi, Phys. Rev. Lett. 114, 050403 (2015).

[24] N. Brunner, D. Cavalcanti, S. Pironio, V. Scarani, and S. Wehner, Rev. Mod. Phys. 86, 419 (2014).

[25] J. Ellis, J. S. Hagelin, D. V. Nanopoulos, and M. Srednicki, Nucl. Phys. B241, 381 (1984).

[26] G. C. Ghirardi, A. Rimini, and T. Weber, Phys. Rev. D 34, 470 (1986).
[27] L. Diosi, Phys. Rev. A 40, 1165 (1989).

[28] R. Penrose, Gen. Relativ. Gravit. 28, 581 (1996).

[29] C. Pfister, J. Kaniewski, M. Tomamichel, A. Mantri, R. Schmucker, N. McMahon, G. Milburn, and S. Wehner, arXiv:1503.00577.

[30] J. F. Clauser, M. Horne, A. Shimony, and R. A. Holt, Phys. Rev. Lett. 23, 880 (1969).

[31] R. F. Werner, Phys. Rev. A 40, 4277 (1989).

[32] V. Scarani, Acta Phys. Slovaca 62, 347 (2012).

[33] J. D. Cohen, S. M. Meenehan, G. S. MacCabe, S. Groblacher, A. H. Safavi-Naeini, F. Marsili, M. D. Shaw, and O. Painter, Nature (London) 520, 522 (2015).

[34] C. Galland, N. Sangouard, N. Piro, N. Gisin, and T. J. Kippenberg, Phys. Rev. Lett. 112, 143602 (2014).

[35] S. G. Hofer, W. Wieczorek, M. Aspelmeyer, and K. Hammerer, Phys. Rev. A 84, 052327 (2011).

[36] V. Caprara Vivoli, P. Sekatski, J.-D. Bancal, C. C. W. Lim, A. Martin, R. T. Thew, H. Zbinden, N. Gisin, and N Sangouard, New J. Phys. 17, 023023 (2015).

[37] K. Banaszek and K. Wodkiewicz, Phys. Rev. Lett. 82, 2009 (1999).

[38] B. Hessmo, P. Usachev, H. Heydari, and G. Björk, Phys. Rev. Lett. 92, 180401 (2004).

[39] A. Kuzmich, I. A. Walmsley, and L. Mandel, Phys. Rev. Lett. 85, 1349 (2000).

[40] F. Monteiro, V. C. Vivoli, T. Guerreiro, A. Martin, J.-D. Bancal, H. Zbinden, R. T. Thew, and N. Sangouard, Phys. Rev. Lett. 114, 170504 (2015).

[41] M. G. A. Paris, Phys. Lett. A 217, 78 (1996).

[42] See Supplemental Material at http://link.aps.org/ supplemental/10.1103/PhysRevLett.116.070405, which includes Refs. [24,31,32,34,43]. Part I gives the joint and marginal probabilities when the mechanical state is not in the vacuum, Part II shows how the locality loophole can be closed.

[43] L. K. Shalm et al., Phys. Rev. Lett. 115, 250402 (2015).

[44] F. Lecocq, J. D. Teufel, J. Aumentado, and R. W. Simmonds, Nat. Phys. 11, 635 (2015).

[45] J. Chan, A. H. Safavi-Naeini, J. T. Hill, S. Meenehan, and O. Painter, Appl. Phys. Lett. 101, 081115 (2012).

[46] E. Kuramochi, H. Taniyama, T. Tanabe, K. Kawasaki, Y.-G. Roh, and M. Notomi, Opt. Express 18, 15859 (2010).

[47] X. Sun, X. Zhang, C. Schuck, and H. X. Tang, Sci. Rep. 3, 1436 (2013).

[48] L.-M. Duan, M. D. Lukin, J. I. Cirac, and P. Zoller, Nature (London) 414, 413 (2001).

[49] S. G. Hofer, K. W. Lehnert, and K. Hammerer, following Letter, Phys. Rev. Lett. 116, 070406 (2016). 